\title{
Inhibition of collagen fibril formation
}

\author{
Andrzej Steplewski, Andrzej Fertala* \\ From Fibroproliferative disorders: from biochemical analysis to targeted therapies \\ Frauenchiemsee, Germany. 25-30 September 2010
}

\begin{abstract}
Background: The overall aim of presented study is to test the inhibition of the formation of collagen fibrils as the novel approach to reduce accumulation of pathological fibrotic deposits. The main hypothesis is that by interfering with the initial steps of the extracellular process of collagen fibril formation, it is possible to reduce the formation of fibrotic tissue.
\end{abstract}

Methods: The experimental model includes antibody-based inhibitors that specifically bind to the sites that participate in the collagen/collagen interaction.

Results: Employed antibody-based inhibitors effectively limit the amount of collagen fibrils formed in vitro and in engineered tissue models of localized fibrosis.

Conclusions: (i) Inhibition of collagen formation is an attractive target to reduce excessive formation of fibrotic tissue. (ii) Antibody-based inhibitors of collagen fibril formation are promising therapeutic agents with a potential to limit localized fibrosis in a number of tissues.

\section{Background \\ Collagen self-assembly}

Collagen I is the most abundant structural protein of connective tissues such as skin, bone, and tendon. This protein is first synthesized as a precursor molecule, procollagen, that is characterized by the presence of a rodlike central triple-helical domain flanked by short linear telopeptides and globular $\mathrm{N}$-terminal and $\mathrm{C}$-terminal propeptides [1]. Single procollagen molecules are the building blocks for the biologically-and mechanicallyrelevant collagen fibrils (Figure 1). The formation of collagen fibrils is initiated by enzymatic cleavage of $\mathrm{N}$ terminal and $\mathrm{C}$-terminal propeptides. The $\mathrm{N}$-terminal propeptides are cleaved by a group of enzymes that includes a disintegrin and metalloprotease with thrombospondin motifs (ADAMTS)-2, -3 , and -14 , while the C-terminal propeptides are processed by the metalloprotease bone morphogenetic protein 1 (BMP-1) and by the other members of a closely related family of mammalian tolloid-like metalloproteases [2-4]. Such removal of procollagen propeptides exposes telopeptides, which

\footnotetext{
* Correspondence: andrzej.fertala@jefferson.edu

Department of Orthopaedic Surgery, Jefferson Medical College, Thomas Jefferson University, Philadelphia, PA, 19107, USA
}

drive collagen self-assembly by engaging in site-specific intermolecular interactions [5] (Figure 1, Figure 2, and Figure 3A).

\section{Collagen and fibrosis}

In physiological conditions homeostasis of tissue collagens is constantly maintained, but during a number of pathological processes, the balance is shifted toward fibrosis, a process of excessive collagen production and accumulation. Fibrosis is a reactive process modulated by various factors propagated by an inflammatory tissue reaction. These factors trigger the local expansion of resident fibroblast subpopulations, modulate anabolic and catabolic processes taking place in these cells, and influence reactions governing the biosynthesis and degradation of the connective tissue components. Contributing to the metabolic modulation of the biosynthesis and degradation of collagenous proteins are cytokines and growth factors, a group of diverse molecules derived from blood cells, such as platelets, or elaborated locally by mesenchymal and epithelial cells [6].

Localized fibrotic reactions are quite common and frequently develop as a consequence of trauma or surgical procedures. For instance, after surgery of the abdomen, 


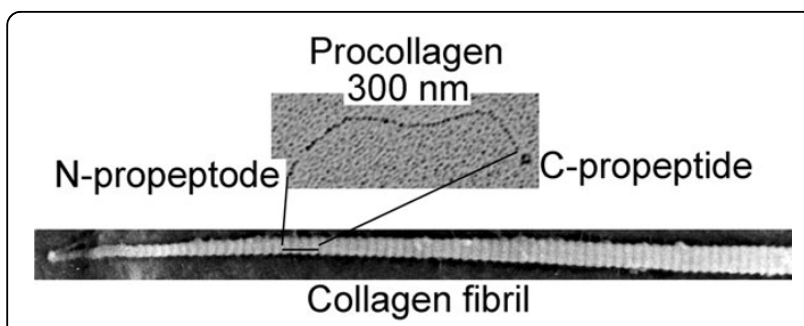

Figure 1 Electron microscopy of a single procollagen molecule and a collagen fibril formed in vitro by self-assembly of collagen molecules. A procollagen molecule, $300 \mathrm{~nm}$ in length, consists of the triple-helical domain flanked with the $\mathrm{N}$ propeptide and the $C$ propeptide. Upon cleavage of the propeptides by specific enzymes, collagen molecules self-assemble to form collagen fibrils.

the formation of excessive scar tissue around abdominal organs, such as the intestines, can interfere with the function of such organs and may cause severe pain and even death. Another situation where excessive scar formation presents a major complication is in the eye after glaucoma surgery performed to create a pressure-maintenance valve. Frequently, however, excessive scar formation closes this pressure-reducing valve, thereby forcing the intraocular pressure to rise [7]. Moreover, excessive scarring of the vocal folds may severely alter their ability to vibrate, thereby causing a number of voice disorders [8].

\section{Inhibitors of fibrosis}

At present, several biological processes critical for the development of fibrotic lesions are considered potential targets for inhibitors of fibrosis. These inhibitors aim at (i) reducing inflammatory processes associated with

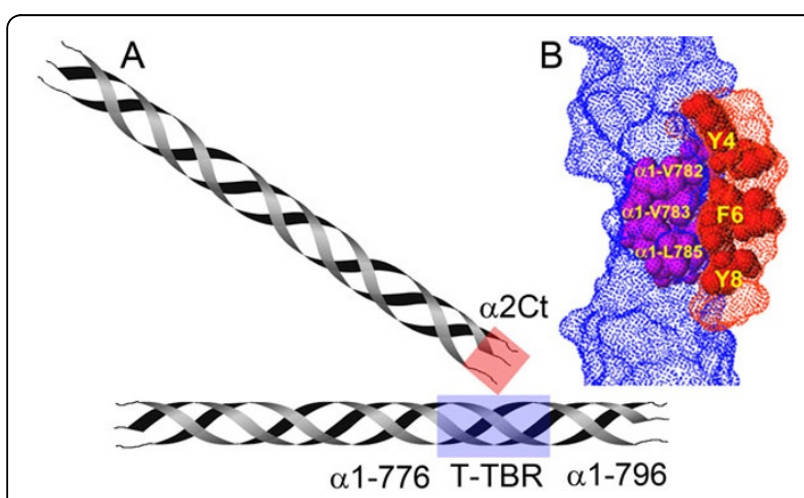

Figure 2 Collagen/collagen interaction sites involved in fibril formation. A, A schematic of collagen molecules interacting during fibril formation. Sites that are involved in collagen/collagen binding are indicated with red and blue boxes. These sites include the T-TBR ( $\alpha 1$ 776-796 fragment) and the $\alpha 2 \mathrm{Ct}$. B, A computer model representing interacting domains of two collagen molecules. The triple-helical fragment of the T-TBR is indicated in blue, while the fragment of the $\alpha 2 \mathrm{Ct}$ of the interacting partner collagen molecule is indicated in red. Amino acid residues most likely engaged in collagen/collagen interaction are also indicated. fibrosis, (ii) inhibiting biological functions of cytokines and growth factors that promote fibrosis, (iii) reducing cell proliferation, and (iv) decreasing the biosynthesis and enzymatic processing of procollagen molecules. The common characteristic of the above approaches is that they target broad upstream processes of the fibrotic cascade. Since most of these processes are involved not only in pathological fibrosis, but also in a number of physiological events, interfering with them is frequently associated with adverse effects [9-13]. Below is a brief characteristic of various clinical and experimental approaches employed currently and in the past to reduce localized fibrotic changes.

\section{Inhibition of the inflammatory phase}

Inhibition of excessive fibrosis at the inflammatory stage has been widely investigated, and at present, is the most established approach to scar management. The inflammatory response can be regulated before, during, and after synthesis of the inflammatory mediators. The most commonly applied anti-inflammatory agents targeting production of inflammatory proteins are glucocorticosteroids. These compounds are effective in some cases of liver and pulmonary fibrosis and also in some patients treated for severe hypertrophic scars of the skin [11,14-17]. Because of the adverse side effects of steroids, particularly when applied systemically, their use is limited in chronic fibrotic diseases. Most nonsteroidal antiinflammatory drugs studied as antifibrotic agents were reported to be ineffective [18].

\section{Inhibition of cytokines}

A number of cytokines and growth factors have been identified in fibrotic processes, and their roles have been associated with the activation of the expression of genes encoding collagens and other structural macromolecules. Among these factors, members of the TGF- $\beta$ family, connective tissue growth factor (CTGF), platelet derived growth factor (PDGF), and epidermal growth factor (EGF) play key roles. Perhaps the most attractive target for inhibiting fibrosis at the level of cytokines is TGF- $\beta 1$. As demonstrated by Shah et al., injecting anti-TGF- $\beta 1$ antibodies into margins of healing wounds significantly decreases scar formation [13]. TGF- $\beta$, however, has normal functions that make chronic administration of any inhibitor that indiscriminately blocks TGF- $\beta$ activity problematic due to unwanted side effects. Moreover, the overall enthusiasm about using an anti-TGF- $\beta 1$ approach to treat fibrosis is hampered by evidence that TGF- $\beta 1$ may also function as a tumor suppressor $[19,20]$. The main concern, then, is that long-term exposure to TGF- $\beta 1$ inhibitors could cause dangerous side-effects in the form of cancers. Still, more recently approaches targeting TGF- $\beta 1$ via blocking connexin-mediated mechanisms were proposed. 


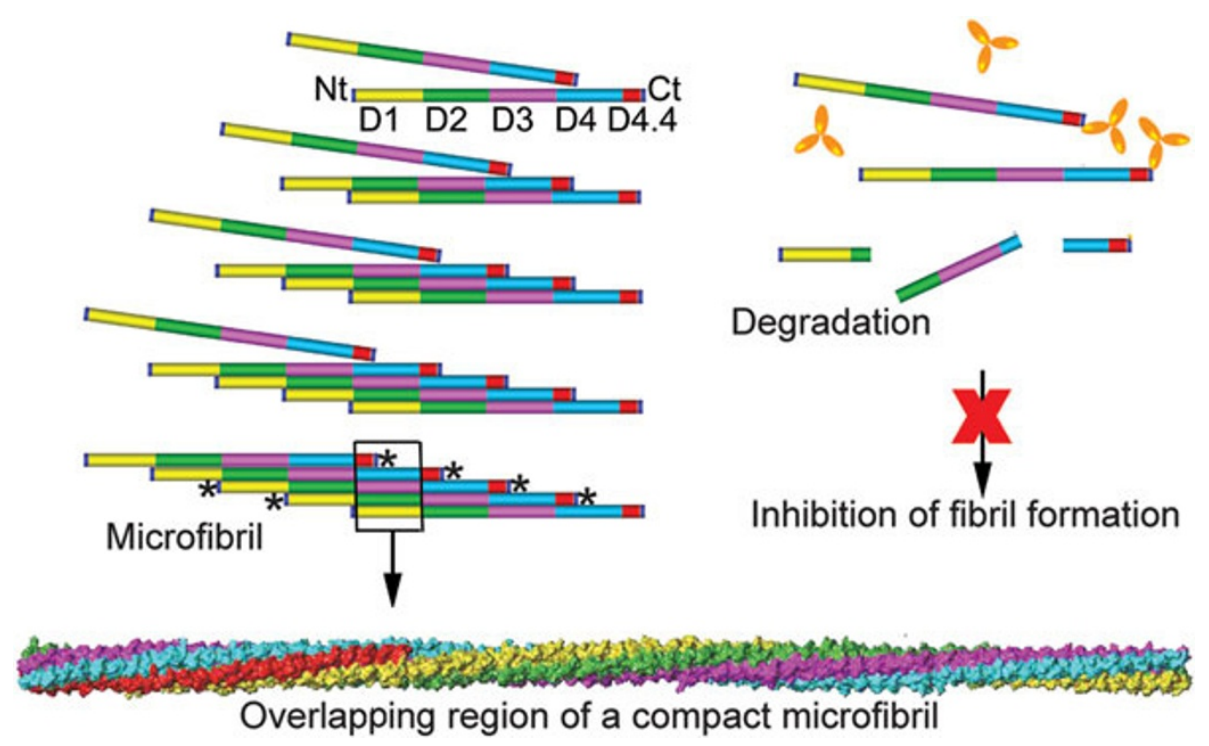

Figure 3 Collagen/collagen interaction sites involved in fibril formation; the basic concept of inhibiting collagen fibril formation. A, the stages of normal fibril formation by site-specific interaction between telopeptides of one collagen molecule and the T-TBR of the interacting molecule located in the D4 period; fibril-incorporated collagen molecules are very resistant to enzymatic degradation. B, by blocking the sites of critical collagen-collagen interaction, formation of fibrils is inhibited, preventing the accumulation of fibrils and allowing rapid degradation of excess collagen molecules. Asterisks represent sites of cross-links formation. The model of a microfibril illustrates the compact packing of collagen molecules (indicated in colors matching those for specific D periods) and indicates how the binding of a bulky inhibitor to collagen molecules would prevent such a compact organization.

Specifically, the studies showed that cell-permeable peptides that block intracellular interactions of connexin 43 alter TGF- $\beta 1$ functions, thereby limiting production of collagen in an animal model [21]. A clinical value of this intracellular approach, however, has not yet been examined.

In addition to targeting TGF- $\beta 1$ and other cytokines at the protein level, attempts were made to inhibit the expression of the corresponding genes by antisense oligonucleotide or RNA interference (RNAi) approaches [22]. Although antisense oligonucleotide technology has been around for about two decades, it appears that, for a variety of reasons, this technology did not find a broad clinical use and it is primarily employed as a research tool.

\section{Inhibition of cell proliferation}

Mitomycin-C and 5-fluorouracil inhibit the proliferation of cells by blocking DNA synthesis and transcription. A single application of these agents by injection was shown to be effective in reducing the recurrence of keloid formation after surgery. Injection of 5-fluorouracil, however, causes considerable pain, thereby limiting the use of this agent for keloid reduction [12,23-25].

\section{Inhibition of collagen synthesis}

Because prolyl-4-hydroxylase-mediated post-translational modifications are required for the correct folding of individual pro- $\alpha$ chains into functional triple-helical molecules and for the secretion of such folded molecules into the extracellular space, this enzyme was identified as a potential target to limit collagen biosynthesis. For instance, "Doxorubicin", a commonly used chemotherapeutic agent that irreversibly inactivates prolyl-4-hydroxylase in human skin fibroblasts, inhibits collagen chain assembly. It is not clear, however, if this agent would be beneficial to patients with excessive scar formation.

Yet another approach to limit the production of "functional" collagen molecules was to administer proline analogues [26]. Because proline is a major residue in fibrillar collagens and constitutes $\sim 10 \%$ of the total pool of collagen amino acid residues, the rationale behind this concept was that incorporating proline analogues to newly synthesized collagen chains, instead of native proline residues, would lead to the formation of nonfunctional collagen molecules. Although a number of proline analogues were developed a few decades ago, to date, they have not been used due to significant toxic side effects [26].

\section{Inhibition of procollagen-processing enzymes}

One of the critical steps that involved in collagen fibril formation is the enzymatic cleavage of procollagen propeptides with procollagen N-proteinase (PNP) and BMP-1 $[27,28]$. The discovery of the amino acid sequence of BMP-1 prompted the research on its potential inhibitors $[4,29,30]$. The rationale of this approach was that 
inhibiting the cleavage of the C-terminal propeptides by BMP-1 would prevent fibril formation, thereby limiting excessive fibrosis. Several inhibitors of BMP-1 (e.g. acidic dipeptide hydroxamate) have been reported to be active in vitro, but their effect on preventing collagen fibril formation is not known [9]. An additional significant problem is that BMP-1, in addition to procollagens, processes a number of other macromolecules central to various important biological events not related to collagen fibril formation [31-35]. Moreover, BMP-1 knockout experiments demonstrated the presence of normal collagen fibrils in tissues of BMP-1-knockout mice, indicating that BMP-1 is not the only enzyme able to process procollagen in vivo, making it an unattractive target for inhibitors of fibrosis [35]. Similarly, PNP knockout experiments demonstrated the presence of fully processed collagen I and collagen II in tissues of experimental mice. Moreover, PNP knockout caused sterility in males, an unexpected adverse effect [36].

\section{Inhibition of formation of fibril-stabilizing cross-links}

In physiological conditions, the chemical cross-linking of collagen molecules incorporated in collagen fibrils is critical for the mechanical stability of fibrils. Moreover, the presence of chemical cross-links makes fibril-incorporated collagen molecules more resistant to proteolysis. This notion is supported by a study which demonstrated that long-term stability of free collagen molecules at $37^{\circ} \mathrm{C}$ is quite low [37]. Because of such instability, exposed individual $\alpha$ chains of these molecules are readily accessible to proteases [37].

Formation of cross-links is an enzymatic process catalyzed by lysyl-oxidase. Since lysyl oxidase is a copperdependent enzyme, it has been proposed that the use of copper chelators, such as D-penicillamine, could result in reduced cross-link formation, thereby limiting tissue fibrosis [38]. Postulations have also been made to employ $\beta$ aminopropionitrile, a compound that inhibits enzymatic activity of lysyl oxidase by irreversible binding to this enzyme [38,39]. In a clinical context, however, inhibitors of lysyl oxidase are not suitable for the treatment of fibrotic diseases because of their considerable toxicity.

\section{Discussion}

\section{A novel concept; limiting tissue fibrosis by direct targeting collagen fibril formation -}

Because of a number of limitations of current approaches to reduce excessive formation of localized fibrotic deposits, Chung et al. have proposed a novel concept. The premise of this new concept is that, regardless of etiology, fibrotic deposits are built primarily from collagen, specifically collagen fibrils. Consequently, Chung et al. have proposed a plan to inhibit formation of fibrotic deposits by limiting formation of collagen fibrils (Figure 2 and Figure 3) [40].
In contrast to the approaches presented above, the method proposed by Chung et al. targets a specific downstream event in a fibrotic cascade. Specifically, in this novel approach, the main target is the collagen/collagen interaction, a key process that drives collagen fibril formation (Figure 2, and Figure 3). The proposed target for inhibiting formation of collagen fibrils is well defined. It has been determined that critical collagen/collagen binding is mediated through interaction of the C-terminal $\alpha 1$ (I) and $\alpha 2$ (I) telopeptides ( $\alpha 1 \mathrm{Ct}$ and $\alpha 2 \mathrm{Ct}$ ) of one collagen molecule and the Triple-helical Telopeptide-Binding Region (T-TBR) of another binding partner (Figure 2). It has been also determined that the T-TBR corresponds to the $\alpha 1$ 776-796 fragment of collagen I (Figure 2) [41]. Note: numbering of amino acid residues considers the first glycine residue in a collagen triple-helical region as number "1".

Even though collagen fibrils in vivo are complex heterotypic structures consisting not only of collagen I, but also of other collagen types, such as collagen III and collagen $\mathrm{V}$, it is predicted that the novel approach targeting collagen I self-assembly will still be effective. This notion is justified because collagen I contributes the most to the total protein mass of fibrotic tissue and other collagen types are most effectively incorporated into a fibril only when collagen I core is present [42]. Thus, by blocking the self-assembly of collagen I, it will be possible to interrupt the entire cascade of heterotypic fibril formation.

The prospect of the novel concept of inhibiting collagen fibril formation by blocking collagen/collagen binding for its clinical utility is supported, in part, by earlier reports on the inhibition of intermolecular interactions. For instance, the formation of amyloid fibrous deposits in Alzheimer's disease can be partially inhibited by a fragment of collagenous Alzheimer amyloid plaque component (CLAC), which blocks binding between full-length CLAC molecules [43]. Moreover, a number of therapeutic agents that act through blocking intermolecular interactions already exist in the clinical practice. Those agents include "Etanercept" (a fusion protein that neutralizes soluble TNF- $\alpha$ in psoriasis, rheumatoid arthritis, and psoriatic arthritis) and "Enfuvirtide" (a synthetic peptide that acts extracellularly and inhibits HIV entry into T cells). These fundamental similarities among approaches to inhibit various intermolecular interactions provide a valid point in support of targeting collagen/collagen interaction to limit excessive formation of collagen deposits.

\section{Strategies of designing inhibitors of collagen fibril formation}

The original concept and the initial results on inhibiting collagen fibril formation as a way to reduce the formation of fibrotic deposits in a keloid-like model were described by Chung et al. [40]. In this study, the authors have demonstrated that a monoclonal antibody that 
binds to the $\alpha 2 \mathrm{C}$-terminal telopeptide of human collagen I (anti- $\alpha 2 \mathrm{Ct}$ ) significantly reduces the amount of collagen deposited in keloid-like constructs formed subcutaneously in nude mice. The authors have concluded that this reduction was a result of blocking the $\alpha 2$ C-terminal telopeptide-mediated collagen/collagen interaction (Figure 2) [40]. Based on the above results, utilizing antibody-based inhibitors is, at present, a leading concept in an approach to reducing localized fibrosis through interfering with collagen fibril formation. Antibody-based approaches include engineering clinicallyrelevant IgG and $\mathrm{scFv}$ variants.

\section{Conclusions}

-Inhibition of collagen formation is an attractive target to reduce excessive formation of fibrotic tissue.

-Unlike canonical inhibitors of excessive accumulation of collagen-rich deposits, inhibition of collagen fibril formation targets a specific, extracellular event.

-Blocking collagen fibril formation may serve as an independent or supporting method to reduce excessive, localized accumulation of collagen-rich deposits.

-Antibody-based inhibitors of collagen fibril formation are promising therapeutic agents with a potential to limit localized fibrosis in a number of tissues.

\section{Acknowledgements}

This article has been published as part of Fibrogenesis \& Tissue Repair Volume 5 Supplement 1, 2012: Proceedings of Fibroproliferative disorders: from biochemical analysis to targeted therapies. The full contents of the supplement are available online at http://www.fibrogenesis.com/ supplements/5/S1.

This work was supported by the National Health Institutes [2R01AR04854406A2 and 1R21AR061118-01].

\section{Authors' contributions}

AS carried out collagen inhibition studies. AF conceived of the study, participated in its design and coordination, and drafted the manuscript. All authors read and approved the final manuscript.

\section{Competing interests}

The authors declare that they have no competing interests.

Published: 6 June 2012

\section{References}

1. Prockop DJ, Kivirikko Kl: Collagens: molecular biology, diseases, and potentials for therapy. Annu Rev Biochem 1995, 64:403-434.

2. Colige A, Vandenberghe I, Thiry M, Lambert CA, Van Beeumen J, Li SW, Prockop DJ, Lapiere CM, Nusgens BV: Cloning and characterization of ADAMTS-14, a novel ADAMTS displaying high homology with ADAMTS2 and ADAMTS-3. J Biol Chem 2002, 277(8):5756-5766.

3. Hopkins DR, Keles S, Greenspan DS: The bone morphogenetic protein 1/ Tolloid-like metalloproteinases. Matrix Biol 2007, 26(7):508-523.

4. Kessler E, Takahara K, Biniaminov L, Brusel M, Greenspan DS: Bone morphogenetic protein-1: the type I procollagen C-proteinase [see comments]. Science 1996, 271(5247):360-362.

5. Kadler KE, Hojima Y, Prockop DJ: Assembly of collagen fibrils de novo by cleavage of the type I pC-collagen with procollagen C-proteinase. Assay of critical concentration demonstrates that collagen self-assembly is a classical example of an entropy-driven process. J Biol Chem 1987, 262(32):15696-15701.
6. Pannu J, Trojanowska M: Recent advances in fibroblast signaling and biology in scleroderma. Curr Opin Rheumatol 2004, 16(6):739-745.

7. Addicks EM, Quigley HA, Green WR, Robin AL: Histologic characteristics of filtering blebs in glaucomatous eyes. Arch Ophthalmol 1983, 101(5):795-798.

8. Lim X, Tateya I, Tateya T, Munoz-Del-Rio A, Bless DM: Immediate inflammatory response and scar formation in wounded vocal folds. Ann Otol Rhinol Laryngol 2006, 115(12):921-929.

9. Ovens A, Joule JA, Kadler KE: Design and synthesis of acidic dipeptide hydroxamate inhibitors of procollagen C-proteinase. J Pept Sci 2000, 6(9):489-495.

10. Riley DJ, Kerr JS, Berg RA, lanni BD, Pietra GG, Edelman NH, Prockop DJ: beta-Aminopropionitrile prevents bleomycin-induced pulmonary fibrosis in the hamster. Am Rev Respir Dis 1982, 125(1):67-73.

11. Roseborough IE, Grevious MA, Lee RC: Prevention and treatment of excessive dermal scarring. J Natl Med Assoc 2004, 96(1):108-116.

12. Sanders KW, Gage-White L, Stucker FJ: Topical mitomycin C in the prevention of keloid scar recurrence. Arch Facial Plast Surg 2005, 7(3):172-175.

13. Shah M, Foreman DM, Ferguson MW: Neutralisation of TGF-beta 1 and TGF-beta 2 or exogenous addition of TGF-beta 3 to cutaneous rat wounds reduces scarring. J Cell Sci 1995, 108(Pt 3):985-1002.

14. Albanis E, Safadi R, Friedman SL: Treatment of hepatic fibrosis: almost there. Curr Gastroenterol Rep 2003, 5(1):48-56.

15. Cohen IK, Diegelmann RF: The biology of keloid and hypertrophic scar and the influence of corticosteroids. Clin Plast Surg 1977, 4(2):297-299.

16. Cohen IK, Diegelmann RF, Johnson ML: Effect of corticosteroids on collagen synthesis. Surgery 1977, 82(1):15-20.

17. Friedman SL, Maher JJ, Bissell DM: Mechanisms and therapy of hepatic fibrosis: report of the AASLD Single Topic Basic Research Conference. Hepatology 2000, 32(6):1403-1408.

18. Friedman SL: Liver fibrosis - from bench to bedside. J Hepatol 2003, 38(Suppl 1):S38-53.

19. Iyer S, Wang ZG, Akhtari M, Zhao W, Seth P: Targeting TGFbeta signaling for cancer therapy. Cancer Biol Ther 2005, 4(3):261-266.

20. Serra R, Crowley MR: Mouse models of transforming growth factor beta impact in breast development and cancer. Endocr Relat Cancer 2005, 12(4):749-760.

21. Rhett JM, Ghatnekar GS, Palatinus JA, O'Quinn M, Yost MJ, Gourdie RG: Novel therapies for scar reduction and regenerative healing of skin wounds. Trends Biotechnol 2008, 26(4):173-180.

22. Jazag A, Kanai F, ljichi H, Tateishi K, Ikenoue T, Tanaka Y, Ohta M, Imamura J, Guleng B, Asaoka Y, et al: Single small-interfering RNA expression vector for silencing multiple transforming growth factor-beta pathway components. Nucleic Acids Res 2005, 33(15):e131.

23. Kelly AP: Medical and surgical therapies for keloids. Dermatol Ther 2004, 17(2):212-218.

24. Poochareon VN, Berman B: New therapies for the management of keloids. J Craniofac Surg 2003, 14(5):654-657.

25. Simman $\mathrm{R}$, Alani $\mathrm{H}$, Williams $\mathrm{F}$ : Effect of mitomycin $\mathrm{C}$ on keloid fibroblasts: an in vitro study. Ann Plast Surg 2003, 50(1):71-76.

26. Riley DJ, Berg RA, Edelman NH, Prockop DJ: Prevention of collagen deposition following pulmonary oxygen toxicity in the rat by cis-4hydroxy-L-proline. J Clin Invest 1980, 65(3):643-651.

27. Holmes DF, Mould AP, Chapman JA: Morphology of sheet-like assemblies of $\mathrm{pN}$-collagen, $\mathrm{pC}$-collagen and procollagen studied by scanning transmission electron microscopy mass measurements. J Mol Biol 1991, 220(1):111-123.

28. Miyahara M, Njieha FK, Prockop DJ: Formation of collagen fibrils in vitro by cleavage of procollagen with procollagen proteinases. $J$ Biol Chem 1982, 257(14):8442-8448.

29. Hartigan N, Garrigue-Antar L, Kadler KE: Bone morphogenetic protein-1 (BMP-1). Identification of the minimal domain structure for procollagen C-proteinase activity. J Biol Chem 2003, 278(20):18045-18049.

30. Li SW, Sieron AL, Fertala A, Hojima Y, Arnold WV, Prockop DJ: The Cproteinase that processes procollagens to fibrillar collagens is identical to the protein previously identified as bone morphogenic protein-1. Proc Natl Acad Sci USA 1996, 93(10):5127-5130.

31. Amano S, Scott IC, Takahara K, Koch M, Champliaud MF, Gerecke DR, Keene DR, Hudson DL, Nishiyama T, Lee S, et al: Bone morphogenetic 
protein 1 is an extracellular processing enzyme of the laminin 5 gamma 2 chain. J Biol Chem 2000, 275(30):22728-22735.

32. Imamura Y, Steiglitz BM, Greenspan DS: Bone morphogenetic protein-1 processes the $\mathrm{NH} 2$-terminal propeptide, and a furin-like proprotein convertase processes the $\mathrm{COOH}$-terminal propeptide of pro-alpha1(V) collagen. J Biol Chem 1998, 273(42):27511-27517.

33. Rattenholl A, Pappano WN, Koch M, Keene DR, Kadler KE, Sasaki T, Timpl R, Burgeson RE, Greenspan DS, Bruckner-Tuderman L: Proteinases of the bone morphogenetic protein-1 family convert procollagen VII to mature anchoring fibril collagen. J Biol Chem 2002, 277(29):26372-26378.

34. Scott IC, Imamura Y, Pappano WN, Troedel JM, Recklies AD, Roughley PJ, Greenspan DS: Bone morphogenetic protein-1 processes probiglycan. J Biol Chem 2000.

35. Suzuki N, Labosky PA, Furuta Y, Hargett L, Dunn R, Fogo AB, Takahara K, Peters DM, Greenspan DS, Hogan BL: Failure of ventral body wall closure in mouse embryos lacking a procollagen C-proteinase encoded by Bmp1, a mammalian gene related to Drosophila tolloid. Development 1996, 122(11):3587-3595.

36. Li SW, Arita M, Fertala A, Bao Y, Kopen GC, Langsjo TK, Hyttinen MM, Helminen HJ, Prockop DJ: Transgenic mice with inactive alleles for procollagen $\mathrm{N}$-proteinase (ADAMTS-2) develop fragile skin and male sterility. Biochem J 2001, 355(Pt 2):271-278.

37. Leikina E, Mertts MV, Kuznetsova N, Leikin S: Type I collagen is thermally unstable at body temperature. Proc Natl Acad Sci USA 2002, 99(3):1314-1318

38. Chang K, Uitto J, Rowold EA, Grant GA, Kilo C, Williamson JR: Increased collagen cross-linkages in experimental diabetes: reversal by betaaminopropionitrile and D-penicillamine. Diabetes 1980, 29(10):778-781.

39. Tang SS, Trackman PC, Kagan HM: Reaction of aortic lysyl oxidase with beta-aminopropionitrile. J Biol Chem 1983, 258(7):4331-4338.

40. Chung HJ, Steplewski A, Chung KY, Uitto J, Fertala A: Collagen fibril formation. A new target to limit fibrosis. J Biol Chem 2008, 283(38):25879-25886.

41. Prockop DJ, Fertala A: Inhibition of the self-assembly of collagen I into fibrils with synthetic peptides. Demonstration that assembly is driven by specific binding sites on the monomers. J Biol Chem 1998, 273(25):15598-15604

42. Romanic AM, Adachi E, Kadler KE, Hojima Y, Prockop DJ: Copolymerization of pNcollagen III and collagen I. pNcollagen III decreases the rate of incorporation of collagen I into fibrils, the amount of collagen I incorporated, and the diameter of the fibrils formed. J Biol Chem 1991, 266(19):12703-12709.

43. Osada Y, Hashimoto T, Nishimura A, Matsuo Y, Wakabayashi T, Iwatsubo T: CLAC binds to amyloid beta peptides through the positively charged amino acid cluster within the collagenous domain 1 and inhibits formation of amyloid fibrils. J Biol Chem 2005, 280(9):8596-8605.

\section{Submit your next manuscript to BioMed Central and take full advantage of:}

- Convenient online submission

- Thorough peer review

- No space constraints or color figure charges

- Immediate publication on acceptance

- Inclusion in PubMed, CAS, Scopus and Google Scholar

- Research which is freely available for redistribution 